\title{
Surgical Castration
}

National Cancer Institute

\section{Source}

National Cancer Institute. Surgical Castration. NCI Thesaurus. Code C15198.

Surgical removal of the ovaries or testes. 